\section{Preço dos alimentos no Brasil: prefira preparações culinárias a alimentos ultraprocessados}

\author{
Food prices in Brazil: prefer cooking to \\ ultra-processed foods
}

\section{Precio de los alimentos en Brasil: elija platos caseros a alimentos ultraprocesados}

Rafael Moreira Claro ${ }^{1}$

Emanuella Gomes Maia 1

Bruna Vieira de Lima Costa 1

Danielle Pereira Diniz 1

\section{Resumo}

Estudo com objetivo de descrever o preço dos grupos de alimentos consumidos no Brasil considerando a natureza, a extensão e o propósito de seu processamento. Dados provenientes da Pesquisa de Orçamentos Familiares de 2008-2009 foram utilizados. O preço médio dos grupos (in natura, ingredientes culinários, processados e ultraprocessados) e seus respectivos subgrupos de alimentos foram estimados para o Brasil segundo renda, região e área. Os produtos in natura e ingredientes culinários apresentaram menor preço por caloria quando comparado aos demais grupos, sugerindo uma vantagem econômica no preparo de refeições no lar em comparação a sua substituição por ultraprocessados. As famílias de maior nível econômico pagaram preço mais elevado por suas aquisições, enquanto as regiões Nordeste, Norte e a área rural pagaram os menores preços. Enquanto alimentos frescos (como carnes, leite, frutas e hortaliças) tendem a custar mais caro que alimentos ultraprocessados, grãos secos (como o arroz e o feijão) despontam como uma alternativa mais econômica para adoção de práticas alimentares saudáveis.

Hábitos Alimentares; Manipulação de Alimentos; Fatores Socioeconômicos

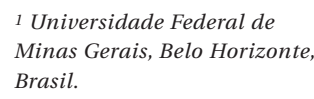

1 Universidade Federal de Minas Gerais, Belo Horizonte, Brasil. 


\section{Introdução}

Nas últimas décadas, a prevalência de obesidade e de doenças crônicas não transmissíveis (DCNT) tem aumentado de forma alarmante, tornandose o principal problema mundial de nutrição em saúde pública 1,2. No Brasil, dados de inquéritos nacionais realizados entre 1975 e 2009 evidenciam esse aumento na prevalência de excesso de peso e obesidade em todas as regiões do país e em todas as classes de renda 3,4,5. Em sentido coincidente, séries históricas de estatísticas de mortalidade disponíveis para as capitais dos estados brasileiros indicam que a proporção de mortes por DCNT aumentou em mais de três vezes entre as décadas de 1930 e 1990 6, sendo grande parte dessas doenças intimamente relacionada à obesidade e aos desequilíbrios na alimentação 5,7.

Uma das causas centrais da epidemia de obesidade e de DCNT é a substituição de alimentos in natura (frescos) e minimamente processados por alimentos ultraprocessados de alta densidade energética e baixa qualidade nutricional. Entretanto, em grande parte dos estudos sobre o consumo alimentar de populações e na confecção de recomendações dietéticas, a natureza, extensão e o propósito do processamento dos alimentos é ignorada ${ }^{8,9}$. Nesse contexto, o Ministério da Saúde do Brasil publicou no ano de 2014 a segunda edição do Guia Alimentar para a População Brasileira, tornando-se o primeiro Guia Alimentar nacional a priorizar o consumo de alimentos frescos (in natura ou minimamente processados) e recomendar a remoção de alimentos ultraprocessados do consumo alimentar cotidiano da população 10 .

Sabe-se que as escolhas alimentares não são determinadas inteiramente por necessidades fisiológicas ou nutricionais, mas também por influência de fatores ambientais, como acessibilidade, disponibilidade, qualidade 11 , publicidade e preço dos alimentos 12,13. Porém, grande parte dessas influências ambientais é amplamente favorável ao consumo de alimentos ultraprocessados 14,15, não dando oportunidade à substituição deles por alimentos frescos ou minimamente processados 14,15,16.

Nesse contexto, ainda que uma série de publicações oficiais do Governo Federal do Brasil tenha reconhecido a importância do preço dos alimentos como facilitadores da adoção de padrões saudáveis de alimentação, como o Plano de Enfrentamento das DCNT (2011-2022) 17, o Plano Intersetorial de Prevenção e Controle da Obesidade 18 e o próprio Guia Alimentar ${ }^{10}$, pouco se sabe sobre o cenário de preço de alimentos no país, levando em conta uma perspectiva propriamente nutricional.
Assim, este estudo objetiva estimar o preço dos grupos de alimentos consumidos nos domicílios brasileiros considerando a natureza, extensão e o propósito do processamento ao qual eles são submetidos, contribuindo para a discussão sobre a adoção das recomendações do Guia Alimentar.

\section{Métodos}

População de estudo e coleta dos dados

Os dados utilizados para este estudo são provenientes da Pesquisa de Orçamentos Familiares (POF) de 2008-2009, realizada pelo Instituto Brasileiro de Geografia e Estatística (IBGE). Trata-se de uma pesquisa de caráter transversal, que visa mensurar as estruturas de consumo, dos gastos e rendimentos das famílias, além de possibilitar o levantamento do perfil das condições de vida da população brasileira 5 .

A POF 2008-2009 utilizou plano amostral complexo por conglomerados, com sorteio dos setores censitários em primeiro estágio e de domicílios, em segundo. Os 12.800 setores do conjunto de setores censitários (Amostra Mestra de Inquéritos Domiciliares) foram agrupados previamente para obter estratos de domicílios com alta homogeneidade geográfica e socioeconômica. Foram consideradas a localização geográfica dos setores (região, unidade da federação, capital ou interior, área urbana ou rural) e a variação do nível econômico das famílias em cada lócus geográfico, com base na renda do indivíduo responsável pelo domicílio (dado obtido no Censo Demográfico de 2000). O número de setores sorteados em cada estrato foi proporcional ao número de domicílios no estrato com pelo menos três setores na amostra de cada estrato. Os domicílios de cada setor foram sorteados por amostragem aleatória simples sem reposição. O número de domicílios com entrevistas, por setor, foi fixado de acordo com a área da pesquisa (12 domicílios nos setores urbanos, 16 nos setores rurais). As entrevistas foram distribuídas uniformemente ao longo de quatro trimestres para reproduzir a variação sazonal de rendimentos e aquisições de alimentos (e de outros produtos) em cada estrato.

Foram utilizadas as aquisições de alimentos e bebidas para consumo domiciliar feitas pela unidade de consumo (famílias) durante um período de sete dias consecutivos. Os registros foram feitos diariamente na Caderneta de Aquisição Coletiva (um dos sete instrumentos de coleta de dados utilizado na POF) por um dos moradores do domicílio ou por entrevistador do IBGE, 
destacando a quantidade, a unidade de medida (com o seu equivalente em peso ou volume), a despesa, o local de compra e a forma de obtenção do produto (monetária ou não monetária) para cada aquisição.

Cada um dos 55.970 domicílios pesquisados pela POF 2008-2009 efetuou, em média, 15,2 aquisições de alimentos e bebidas - resultando em um total aproximado de 850 mil aquisições de alimentos e bebidas para consumo domiciliar - divididas entre cerca de 1.500 produtos. $\mathrm{O}$ valor pago em cada aquisição foi, ao término do estudo, deflacionado pelo IBGE para a data referencial da POF 2008-2009 (15 de janeiro de 2009).

O curto período de referência empregado para o registro das despesas com alimentação em cada domicílio não permite que se conheça o padrão usual de aquisição de alimentos de cada domicílio estudado. Assim, optou-se por utilizar, como unidade de estudo, agregados de domicílios correspondentes aos domicílios estudados em cada um dos 550 estratos amostrais que garantissem unidades de estudo com considerável amplitude de variação geográfica e socioeconômica, cujo padrão de aquisição anual de alimentos pode ser conhecido com precisão.

\section{Criação e descrição de variáveis}

Inicialmente, os registros de cada um dos cerca de 1.500 alimentos adquiridos pelas famílias de um mesmo estrato, ao longo de sete dias, foram somados. Tabelas de composição de alimentos (preferencialmente, a Tabela Brasileira de Composição de Alimentos - TACO, versão 4.1 19, complementada pela tabela de composição de alimentos oficial dos Estados Unidos 20) foram utilizadas no intuito de excluir a fração não comestível dos alimentos e converter as quantidades adquiridas de alimentos em seu equivalente energético ( $\mathrm{kcal}$ ). As despesas e as quantidades de cada aquisição foram divididas por sete para expressar valores diários de gasto e de quantidades adquiridas.

A classificação de alimentos proposta no Guia Alimentar para a População Brasileira 10, detalhada por Monteiro et al. 21, foi utilizada para organização dos alimentos. Desse modo, os alimentos foram divididos em três grandes grupos: (a) alimentos in natura ou minimamente processados (frescos); (b) ingredientes para uso culinário (ou na indústria de alimentos); e (c) alimentos processados e ultraprocessados. Os alimentos foram ainda classificados em subgrupos dentro de cada um desses grupos.

O preço pago pelas famílias para os grupos e subgrupos de alimentos foi expresso em reais por unidade de energia fornecida ( $\mathrm{R} \$ 1.000 \mathrm{k}$ - cal), de forma a contextualizá-lo em perspectiva propriamente nutricional 13 . Tal artifício é importante em estudos dessa natureza, pois permite comparar facilmente o preço de dietas isocalóricas compostas pelos diversos grupos de alimentos. Esse preço foi obtido pela divisão da somatória da despesa de cada uma das famílias com a aquisição de alimentos pertencentes ao grupo (ou subgrupo), pela somatória das calorias provenientes de alimentos desse grupo (ou subgrupo). Em seguida, o resultado dessa divisão foi multiplicado por 1.000 (tal multiplicação objetiva apenas facilitar a compreensão e comparação dos preços). No caso de famílias que não adquiriram um determinado grupo de alimentos, os preços foram imputados. Para essa imputação, utilizou-se a mediana do preço pago por famílias que estivessem no mesmo quintil de gastos totais per capita, no mesmo lócus geográfico, e que tivessem sido estudadas no mesmo trimestre do ano (por exemplo, famílias no quintil superior de renda, morando no Município do Rio de Janeiro, estudadas no primeiro trimestre de realização da POF). Esse procedimento faz-se necessário para compor um cenário de escolha de não aquisição frente a um valor de preço superior àquele que a família intentava pagar. Alimentos isentos de calorias como água, café, chá e infusões não tiveram seus preços calculados.

\section{Análise de dados}

O preço médio dos grupos e subgrupos de alimentos (e seu intervalo de 95\% de confiança IC95\%) foi estimado para o conjunto completo da amostra estudada e segundo estratos definidos de acordo com o nível de renda per capita (quintos da distribuição de renda domiciliar per capita), pelas cinco grandes regiões geográficas (Norte, Nordeste, Centro-oeste, Sudeste e Sul) e por área (urbana ou rural) em que o domicílio estava situado.

A identificação de diferença significativa entre o preço dos grupos e subgrupos de alimentos foi feita com comparação de seus intervalos de confiança. Em casos em que não fosse verificada sobreposição dos intervalos, considerou-se a diferença como significativa ( $\mathrm{p} \leq 0,05)$.

A organização do banco e as análises foram realizadas com a utilização do software Stata versão 12.1 (StataCorp LP, College Station, Estados Unidos).

\section{Resultados}

Os grupos de alimentos in natura e minimamente processados, e de alimentos processa- 
dos e ultraprocessados apresentaram os preços mais elevados ( $\mathrm{R} \$ / 1.000 \mathrm{kcal})$, respectivamente, $\mathrm{R} \$ 2,28$ e $\mathrm{R} \$ 2,40$ (sem diferença estatisticamente significante entre eles). Já o grupo com menor preço por caloria foi o de ingredientes culinários, R\$ 0,64 (Tabela 1).

Tabela 1

Preço médio (R $\$ 1.000 \mathrm{kcal})$ pago por grupos e subgrupos de alimentos definidos segundo natureza, extensão e o propósito do processamento aos quais eles foram submetidos. Pesquisa de Orçamentos Familiares, Brasil, 2008-2009.

\begin{tabular}{|c|c|c|}
\hline \multirow[t]{2}{*}{ Grupos e subgrupos de alimentos } & \multicolumn{2}{|c|}{ Preço dos alimentos (R $\$ 1.000 \mathrm{kcal})$} \\
\hline & Média & IC95\% \\
\hline In natura e minimamente processados (1) & 2,28 & $2,21-2,34$ \\
\hline Arroz & 0,53 & $0,53-0,54$ \\
\hline Feijão & 0,98 & $0,96-0,99$ \\
\hline Carnes & 4,38 & $4,27-4,48$ \\
\hline Leite & 2,52 & $2,48-2,56$ \\
\hline Frutas & 4,17 & $4,08-4,26$ \\
\hline Raízes e tubérculos & 2,31 & $2,25-2,36$ \\
\hline Hortaliças & 10,34 & $10,15-10,53$ \\
\hline Peixes & 8,46 & $8,01-8,90$ \\
\hline Ovos & 3,16 & $3,10-3,22$ \\
\hline Outros alimentos & 3,09 & $2,90-3,27$ \\
\hline Ingredientes culinários (2) & 0,64 & $0,63-0,66$ \\
\hline Açúcar & 0,28 & $0,27-0,28$ \\
\hline Óleos vegetais & 0,39 & $0,37-0,40$ \\
\hline Farinha de mandioca & 0,48 & $0,47-0,49$ \\
\hline Farinha de trigo & 0,56 & $0,54-0,57$ \\
\hline Macarrão & 0,93 & $0,91-0,95$ \\
\hline Gordura animal & 1,58 & $1,52-1,65$ \\
\hline Outros ingredientes & 6,06 & $4,88-7,24$ \\
\hline In natura e minimamente processados e ingredientes culinários em conjunto $(1+2)$ & 1,56 & $1,51-1,61$ \\
\hline Processados e ultraprocessados & 2,40 & $2,33-2,46$ \\
\hline Processados & 3,88 & $3,75-4,01$ \\
\hline Queijos & 3,96 & $3,87-4,04$ \\
\hline Carnes processadas & 3,85 & $3,63-4,08$ \\
\hline Conservas & 5,12 & $4,76-5,48$ \\
\hline Ultraprocessados & 2,26 & $2,20-2,33$ \\
\hline Pães & 1,48 & $1,46-1,51$ \\
\hline Produtos doces ou sobremesas & 1,99 & $1,94-2,04$ \\
\hline Produtos de panificação & 1,66 & $1,62-1,69$ \\
\hline Confeitos e outros produtos à base de açúcar & 2,57 & $2,50-2,63$ \\
\hline Salgadinhos e petiscos salgados & 1,46 & $1,42-1,49$ \\
\hline Bebidas adoçadas & 5,29 & $5,20-5,38$ \\
\hline Refrigerantes & 4,15 & $4,07-4,23$ \\
\hline Outras bebidas & 9,64 & $9,34-9,94$ \\
\hline Carnes ultraprocessadas & 2,37 & $2,32-2,43$ \\
\hline Pratos ou refeições prontas & 3,15 & $3,05-3,25$ \\
\hline Molhos e patês & 1,71 & $1,65-1,77$ \\
\hline Cereais matinais & 2,43 & $2,25-2,61$ \\
\hline Bebidas alcoólicas & 6,72 & $6,48-6,96$ \\
\hline Total & 1,78 & $1,73-1,84$ \\
\hline
\end{tabular}

IC95\%: intervalo de 95\% de confiança. 
De modo semelhante, entre os subgrupos de alimentos, os preços mais elevados foram encontrados dentro dos grupos de alimentos in natura e minimamente processados e de alimentos processados e ultraprocessados, destacando-se as hortaliças ( $R$ \$ 10,34), os peixes frescos $(R \$ 8,46)$ e bebidas alcoólicas ( $R \$ 6,72)$, seguidos por bebidas adoçadas ( $\mathrm{R} \$ 5,29)$, conservas ( $\mathrm{R} \$ 5,12) \mathrm{e}$ carne fresca (R\$4,38). Já os menores preços foram verificados para subgrupos dos ingredientes culinários, com menor preço médio para o açúcar (R\$ 0,28), óleos vegetais ( $\mathrm{R} \$ 0,39)$ e farinha de mandioca $(\mathrm{R} \$ 0,48)$ (Tabela 1$)$.

O preço médio resultante da associação dos alimentos in natura e minimamente processados aos ingredientes culinários, condição necessária para o preparo dos alimentos, foi de $\mathrm{R} \$$ 1,56 . Enquanto a média do preço verificada para os alimentos processados e ultraprocessados foi $\mathrm{R} \$ 2,40$. Essa diferença foi estatisticamente significativa, sugerindo uma vantagem econômica no preparo de refeições no lar em comparação a sua substituição por refeições prontas e produtos alimentícios industrializados (Tabela 1).

Observa-se que as famílias de maior nível econômico tenderam a pagar valor mais elevado por suas aquisições quando comparadas às famílias mais pobres. Tal situação foi verificada para todos os grupos e subgrupos de alimentos pesquisados. Isso resultou em que a razão entre o preço pago pelo conjunto dos alimentos in natura e minimamente processados e ingredientes culinários e aquele pago pelos alimentos processados e ultraprocessados se mantivesse estável (Tabela 2). De forma geral, em qualquer estrato de renda, o preço de uma caloria de alimento processado ou ultraprocessado equivale ao preço de cerca de 1,5 caloria do conjunto dos demais alimentos.

Seguindo a tendência observada entre os estratos de renda da população, menores preços foram encontrados nas regiões de menor renda do país - Norte e Nordeste - e na área rural (Tabelas 3, 4 e 5). A razão entre valores pagos pelos alimentos processados e ultraprocessados e aqueles pagos pelos alimentos in natura e minimamente processados foi maior na Região Centro-oeste $(1,80)$ e menor na Nordeste $(1,38)$. Já com relação às áreas urbana e rural, a última apresentou maior razão $(1,73$ vs. 1,51).

\section{Discussão}

O registro detalhado da aquisição de alimentos de mais de 55 mil famílias brasileiras, realizado pela POF 2008-2009, possibilitou estimar o preço dos principais grupos de alimentos consu- midos no Brasil segundo natureza, propósito e intensidade de seu processamento 22, de acordo recomendações da nova edição (2014) do Guia Alimentar para População Brasileira 10. O valor médio dos alimentos ultraprocessados foi maior do que aquele verificado para o conjunto dos demais alimentos, demonstrando que a vantagem econômica para aquisição desses alimentos observada nos Estados Unidos 15 ou no Reino Unido ${ }^{15}$, não é verificada no Brasil. Essa relação se manteve constante em todos os grupos de renda, nas grandes regiões geográficas e no estrato urbano e rural do país. Nossos resultados indicam a possibilidade de que, em cenários de preços semelhantes ao nosso estudo, as recomendações do Guia Alimentar possam ser adotadas sem prejuízo ao custo total da dieta, desde que alimentos processados e ultraprocessados sejam substituídos por alimentos in natura ou minimamente processados de baixo preço por unidade energética (R\$/kcal), como exemplo, os cereais e leguminosas.

Importa lembrar que os resultados do presente estudo se referem exclusivamente à aquisição de alimentos e bebidas para consumo no domicílio (e não ao seu consumo efetivo). No Brasil, nos anos de 2008-2009, os alimentos e bebidas adquiridos para consumo no domicílio respondiam por cerca de $70 \%$ das despesas totais com alimentação das famílias nas regiões metropolitanas 5. Assim, uma vez que este estudo se destina exclusivamente a estimar e comparar o preço dos alimentos adquiridos pela população brasileira, acredita-se que tal fração das aquisições analisadas seja suficiente para fornecer informações com boa validade.

Destaca-se também que dados sobre aquisição de alimentos, semelhantes aos utilizados neste estudo, têm sido usados como uma das principais fontes de informação para estudos sobre alimentação e seus determinantes 23,24. Dados de pesquisas de orçamentos familiares refletem o início da cadeia doméstica de consumo e permitem estabelecer políticas públicas que podem modificar a oferta de alimentos e os padrões de compra da população.

Apesar de o preço dos alimentos aferidos em nosso estudo não apresentar quadro favorável ao consumo dos ultraprocessados (como em alguns países desenvolvidos 15), ele já responde por cerca de uma a cada três calorias consumidas pela população brasileira $(27,8 \%)$ 9. Esse cenário possivelmente se deve à influência de outros determinantes do consumo alimentar, como a alta conveniência e o grande apelo publicitário envolvidos em seu consumo ${ }^{25}$. Ainda que os resultados desta pesquisa não avaliem a tendência temporal na variação do preço dos alimentos, 
Tabela 2

Preço médio $(\mathrm{R} \$ / 1.000 \mathrm{kcal})$ pago por grupos e subgrupos de alimentos definidos segundo natureza, extensão e o propósito do processamento aos quais eles foram submetidos, segundo níveis de renda per capita. Pesquisa de Orçamentos Familiares, Brasil, 2008-2009.

\section{Grupos e subgrupos de alimentos}

Preço dos alimentos (R\$1.000kcal)

Quintos crescentes da distribuição de renda per capita

Média

In natura e minimamente processados (1)

Arroz

Feijão

Carnes

Leite

Frutas

Raízes e tubérculos

Hortaliças

Peixes

Ovos

Outros alimentos

Ingredientes culinários (2)

Açúcar

Óleos vegetais

Farinha de mandioca

Farinha de trigo

Macarrão

Gordura animal

Outros ingredientes

In natura e minimamente processados e ingredientes culinários em conjunto $(1+2)$

Processados e ultraprocessados

Processados

Queijos

Carnes processadas

Conservas

Ultraprocessados

Pães

Produtos doces ou sobremesas

Produtos de panificação

Confeitos e outros produtos à base de açúcar

Salgadinhos e petiscos salgados

Bebidas adoçadas

Refrigerantes

Outras bebidas

Carnes ultraprocessadas

Pratos ou refeições prontas

Molhos e patês

Cereais matinais

Bebidas alcoólicas
1으

IC95\%

IC95\% Média

(C95\%

3으

1,82

0,54

0,90

3,70

2,24

3,43

2,26

9,79

5,81

3,59

2,42

0,53

0,28

0,36

0,42

0,66

0,87

1,30

2,44

1,19

1,85

3,59

3,69

4,07

2,94

1,69

1,30

1,62

1,39

2,41

1,21

5,33

4,17

11,37

2,01

2,54

1,27

1,69

5,47

1,30
1,75-1,89

0,53-0,55

0,87-0,93

3,61-3,79

2,13-2,34

$3,26-3,60$

2,09-2,43

9,53-10,04

$5,51-6,11$

$3,49-3,70$

2,10-2,75

0,52-0,54

0,28-0,29

$0,36-0,37$

0,41-0,43

0,64-0,68

0,85-0,89

1,22-1,39

2,03-2,84

$1,16-1,23$

1,79-1,91

$3,39-3,80$

3,51-3,87

3,79-4,35

2,57-3,32

1,63-1,74

1,26-1,33

$1,56-1,68$

1,34-1,44

$2,32-2,50$

1,18-1,25

5,17-5,50

4,04-4,29

$10,32-12,42$

$1,96-2,07$

2,40-2,67

$1,17-1,36$

1,49-1,89

4,97-5,98

1,26-1,33

\section{2,25}

0,52

0,99

4,40

2,51

4,21

2,30

10,44

8,28

2,92

3,02

0,61

0,27

$$
0,34
$$

0,49

0,52

0,93

1,50

6,03

1,56

2,42

3,50

3,87

3,15

5,13

2,33

1,51

2,02

1,72

2,46

1,50

5,03

3,94

9,33

2,43

3,14

1,83

2,78

6,84

1,80
IC95\%

2,12-2,39

0,51-0,53

0,96-1,02

4,20-4,61

2,44-2,58

4,08-4,33

2,23-2,38

$10,03-10,84$

7,61-8,95

2,83-3,00

2,53-3,51

0,59-0,63

$0,26-0,28$

$0,32-0,37$

0,47-0,50

$0,50-0,53$

0,90-0,95

$1,30-1,69$

4,86-7,19

1,46-1,66

2,34-2,50

$3,21-3,79$

$3,64-4,10$

2,55-3,75

4,31-5,95

2,24-2,41

1,48-1,55

1,94-2,09

1,65-1,79

2,35-2,56

$1,42-1,58$

4,89-5,17

3,80-4,08

$8,82-9,85$

2,36-2,51

2,94-3,35

1,70-1,95

2,28-3,29

$6,10-7,58$

$1,70-1,89$
5 으

Média

2,97

0,55

1,02

5,30

2,86

4,94

2,49

11,43

11,77

3,21

4,08

0,86

0,30

0,51

0,50

0,50

1,02

1,84

8,74

2,09

4,76

4,52

4,66

7,01

2,77

1,71

2,35

1,88

2,87

1,67

5,69

4,38

8,75

2,76

3,84

2,03

2,54

7,41

IC95\%

2,79-3,15

0,53-0,57

0,98-1,07

5,11-5,49

2,80-2,92

4,73-5, 15

2,36-2,63

$10,95-11,91$

$10,39-13,16$

3,08-3,35

$3,67-4,50$

$0,80-0,93$

0,29-0,32

0,44-0,58

0,48-0,52

0,48-0,53

0,96-1,09

1,74-1,95

7,13-10,34

$1,95-2,23$

$2,96 \quad 2,85-3,08$

$4,55-4,96$

4,37-4,68

$4,21-5,11$

2,40 
Tabela 3

Preço médio $(\mathrm{R} \$ / 1.000 \mathrm{kcal})$ pago por grupos e subgrupos de alimentos definidos segundo natureza, extensão e o propósito do processamento aos quais eles foram submetidos, para regiões Norte e Nordeste. Pesquisa de Orçamentos Familiares, Brasil, 2008-2009.

\begin{tabular}{|c|c|c|c|c|}
\hline \multirow[t]{3}{*}{ Grupos e subgrupos de alimentos } & \multicolumn{4}{|c|}{ Preço dos alimentos (R $\$ 1.000 \mathrm{kcal})$} \\
\hline & \multicolumn{2}{|c|}{ Norte } & \multicolumn{2}{|c|}{ Nordeste } \\
\hline & Média & IC95\% & Média & IC95\% \\
\hline In natura e minimamente processados (1) & 2,21 & $2,08-2,35$ & 2,09 & $2,01-2,17$ \\
\hline Arroz & 0,57 & $0,55-0,58$ & 0,55 & $0,54-0,55$ \\
\hline Feijão & 1,10 & $1,06-1,13$ & 0,90 & $0,88-0,92$ \\
\hline Carnes & 3,53 & $3,42-3,64$ & 3,85 & $3,76-3,94$ \\
\hline Leite & 2,52 & $2,25-2,79$ & 2,39 & $2,31-2,46$ \\
\hline Frutas & 3,94 & $3,40-4,48$ & 3,63 & $3,50-3,75$ \\
\hline Raízes e tubérculos & 2,31 & $1,93-2,68$ & 2,36 & $2,25-2,46$ \\
\hline Hortaliças & 11,10 & $10,72-11,48$ & 9,67 & $9,46-9,88$ \\
\hline Peixes & 5,39 & $4,83-5,95$ & 6,69 & $6,41-6,97$ \\
\hline Ovos & 3,72 & $3,61-3,82$ & 3,56 & $3,46-3,66$ \\
\hline Outros alimentos & 3,36 & $2,65-4,08$ & 2,65 & $2,31-2,98$ \\
\hline Ingredientes culinários processados (2) & 0,56 & $0,54-0,59$ & 0,58 & $0,57-0,60$ \\
\hline Açúcar & 0,31 & $0,30-0,32$ & 0,28 & $0,27-0,28$ \\
\hline Óleos vegetais & 0,36 & $0,34-0,37$ & 0,39 & $0,38-0,41$ \\
\hline Farinha de mandioca & 0,44 & $0,42-0,46$ & 0,42 & $0,41-0,44$ \\
\hline Farinha de trigo & 0,66 & $0,64-0,69$ & 0,67 & $0,65-0,69$ \\
\hline Macarrão & 0,94 & $0,92-0,96$ & 0,86 & $0,84-0,88$ \\
\hline Gordura animal & 1,40 & $1,30-1,50$ & 1,46 & $1,38-1,53$ \\
\hline Outros ingredientes & 3,19 & $2,83-3,55$ & 2,45 & $2,11-2,80$ \\
\hline In natura e minimamente processados e ingredientes culinários em conjunto $(1+2)$ & 1,44 & $1,36-1,51$ & 1,39 & $1,34-1,45$ \\
\hline Processados e ultraprocessados & 2,30 & $2,21-2,38$ & 1,93 & $1,88-1,99$ \\
\hline Processados & 4,15 & $3,92-4,37$ & 3,86 & $3,71-4,01$ \\
\hline Queijos & 3,69 & $3,47-3,90$ & 3,89 & $3,75-4,03$ \\
\hline Carnes processadas & 4,59 & $4,30-4,88$ & 4,32 & $4,12-4,52$ \\
\hline Conservas & 4,28 & $3,85-4,70$ & 2,85 & $2,57-3,12$ \\
\hline Ultraprocessados & 2,15 & $2,06-2,24$ & 1,74 & $1,69-1,79$ \\
\hline Pães & 1,40 & $1,37-1,44$ & 1,26 & $1,23-1,28$ \\
\hline Produtos doces ou sobremesas & 1,97 & $1,91-2,03$ & 1,69 & $1,63-1,74$ \\
\hline Produtos de panificação & 1,65 & $1,60-1,70$ & 1,44 & $1,40-1,48$ \\
\hline Confeitos e outros produtos à base de açúcar & 2,56 & $2,48-2,65$ & 2,49 & $2,40-2,58$ \\
\hline Salgadinhos e petiscos salgados & 1,33 & $1,28-1,39$ & 1,20 & $1,17-1,22$ \\
\hline Bebidas adoçadas & 5,68 & $5,40-5,96$ & 5,47 & $5,33-5,61$ \\
\hline Refrigerantes & 4,65 & $4,43-4,43$ & 4,38 & $4,26-4,51$ \\
\hline Outras bebidas & 10,29 & $9,54-11,04$ & 10,44 & $9,58-11,29$ \\
\hline Carnes ultraprocessadas & 2,32 & $2,21-2,44$ & 2,14 & $2,07-2,20$ \\
\hline Pratos ou refeições prontas & 3,08 & $2,86-3,29$ & 2,79 & $2,64-2,94$ \\
\hline Molhos e patês & 1,37 & $1,26-1,48$ & 1,29 & $1,21-1,36$ \\
\hline Cereais matinais & 2,40 & $1,92-2,88$ & 1,56 & $1,40-1,72$ \\
\hline Bebidas alcoólicas & 7,33 & $6,82-7,84$ & 5,77 & $5,33-6,21$ \\
\hline Total & 1,59 & $1,51-1,68$ & 1,51 & $1,46-1,57$ \\
\hline
\end{tabular}

IC95\%: intervalo de 95\% de confiança. 
Tabela 4

Preço médio $(\mathrm{R} \$ / 1.000 \mathrm{kcal})$ pago por grupos e subgrupos de alimentos definidos segundo natureza, extensão e o propósito do processamento aos quais eles foram submetidos, para regiões Sudeste, Sul e Centro-oeste. Pesquisa de Orçamentos Familiares, Brasil, 2008-2009.

\begin{tabular}{|c|c|c|c|c|c|c|}
\hline \multirow[t]{3}{*}{ Grupos e subgrupos de alimentos } & \multicolumn{6}{|c|}{ Preço dos alimentos $(\mathrm{R} \$ / 1.000 \mathrm{kcal})$} \\
\hline & \multicolumn{2}{|c|}{ Sudeste } & \multicolumn{2}{|c|}{ Sul } & \multicolumn{2}{|c|}{ Centro-oeste } \\
\hline & Média & IC95\% & Média & IC95\% & Média & IC95\% \\
\hline In natura e minimamente processados (1) & 2,38 & $2,26-2,50$ & 2,49 & $2,35-2,63$ & 2,00 & $1,85-2,15$ \\
\hline Arroz & 0,54 & $0,53-0,55$ & 0,50 & $0,49-0,51$ & 0,51 & $0,49-0,52$ \\
\hline Feijão & 0,98 & $0,96-1,00$ & 1,08 & $1,021,13$ & 0,93 & $0,89-0,97$ \\
\hline Carnes & 4,79 & $4,63-4,94$ & 4,45 & $4,27-4,63$ & 4,38 & $4,15-4,61$ \\
\hline Leite & 2,62 & $2,56-2,68$ & 2,45 & $2,37-2,53$ & 2,53 & $2,44-2,63$ \\
\hline Frutas & 4,51 & $4,37-4,66$ & 4,09 & $3,94-4,24$ & 4,43 & $4,20-4,67$ \\
\hline Raízes e tubérculos & 2,41 & $2,33-2,48$ & 1,93 & $1,86-2,00$ & 2,30 & $2,19-2,42$ \\
\hline Hortaliças & 10,51 & $10,17-10,86$ & 10,09 & $9,73-10,46$ & 11,43 & $10,98-11,88$ \\
\hline Peixes & 9,07 & $8,43-9,72$ & 11,11 & $9,2-12,966$ & 8,35 & $7,28-9,43$ \\
\hline Ovos & 2,97 & $2,89-3,05$ & 2,78 & $2,71-2,85$ & 3,17 & $3,06-3,28$ \\
\hline Outros alimentos & 3,50 & $3,16-3,85$ & 2,53 & $2,33-2,74$ & 3,09 & $2,73-3,45$ \\
\hline Ingredientes culinários processados (2) & 0,68 & $0,64-0,72$ & 0,70 & $0,66-0,74$ & 0,61 & $0,57-0,64$ \\
\hline Açúcar & 0,28 & $0,27-0,29$ & 0,29 & $0,28-0,30$ & 0,27 & $0,26-0,27$ \\
\hline Óleos vegetais & 0,40 & $0,37-0,44$ & 0,36 & $0,33-0,39$ & 0,34 & $0,32-0,36$ \\
\hline Farinha de mandioca & 0,50 & $0,48-0,51$ & 0,51 & $0,49-0,54$ & 0,50 & $0,48-0,53$ \\
\hline Farinha de trigo & 0,53 & $0,51-0,55$ & 0,41 & $0,40-0,42$ & 0,53 & $0,51-0,55$ \\
\hline Macarrão & 0,94 & $0,90-0,97$ & 1,01 & $0,99-1,04$ & 0,93 & $0,90-0,96$ \\
\hline Gordura animal & 1,65 & $1,53-1,77$ & 1,61 & $1,45-1,78$ & 1,71 & $1,52-1,89$ \\
\hline Outros ingredientes & 8,83 & $6,22-11,44$ & 5,38 & $4,46-6,31$ & 6,32 & $5,42-7,22$ \\
\hline $\begin{array}{l}\text { In natura e minimamente processados e ingredientes culinários em } \\
\text { conjunto }(1+2)\end{array}$ & 1,66 & $1,57-1,75$ & 1,66 & $1,56-1,76$ & 1,45 & $1,34-1,56$ \\
\hline Processados e ultraprocessados & 2,58 & $2,47-2,68$ & 2,60 & $2,52-3,69$ & 2,62 & $2,50-2,73$ \\
\hline Processados & 3,90 & $3,63-4,16$ & 3,91 & $3,71-4,11$ & 3,57 & $3,32-3,82$ \\
\hline Queijos & 4,17 & $4,04-4,31$ & 3,73 & $3,57-3,90$ & 3,65 & $3,48-3,82$ \\
\hline Carnes processadas & 3,54 & $3,10-3,98$ & 3,84 & $3,49-4,19$ & 3,46 & $3,01-3,91$ \\
\hline Conservas & 6,28 & $5,60-6,96$ & 5,89 & $5,38-6,40$ & 5,43 & $4,78-6,07$ \\
\hline Ultraprocessados & 2,46 & $2,36-2,56$ & 2,50 & $2,42-2,58$ & 2,55 & $2,44-2,66$ \\
\hline Pães & 1,60 & $1,56-1,63$ & 1,56 & $1,52-1,60$ & 1,55 & $1,51-1,58$ \\
\hline Produtos doces ou sobremesas & 2,07 & $1,98-2,17$ & 2,23 & $2,17-2,29$ & 2,09 & $1,99-2,18$ \\
\hline Produtos de panificação & 1,73 & $1,67-1,79$ & 1,81 & $1,74-1,87$ & 1,70 & $1,64-1,76$ \\
\hline Confeitos e outros produtos à base de açúcar & 2,56 & $2,43-2,69$ & 2,72 & $2,61-2,82$ & 2,56 & $2,40-2,72$ \\
\hline Salgadinhos e petiscos salgados & 1,56 & $1,50-1,62$ & 1,64 & $1,59-1,70$ & 1,51 & $1,46-1,56$ \\
\hline Bebidas adoçadas & 5,20 & $5,05-5,36$ & 4,99 & $4,80-5,19$ & 5,46 & $5,28-5,64$ \\
\hline Refrigerantes & 3,97 & $3,84-4,11$ & 3,98 & $3,85-4,11$ & 4,28 & $4,15-4,40$ \\
\hline Outras bebidas & 9,31 & $8,90-9,71$ & 8,71 & $8,33-9,08$ & 10,16 & $9,44-10,87$ \\
\hline Carnes ultraprocessadas & 2,49 & $2,40-2,58$ & 2,42 & $2,33-2,50$ & 2,47 & $2,33-2,60$ \\
\hline Pratos ou refeições prontas & 3,36 & $3,18-3,54$ & 3,18 & $3,03-3,33$ & 3,16 & $2,97-3,36$ \\
\hline Molhos e patês & 1,96 & $1,87-2,04$ & 1,80 & $1,73-1,87$ & 1,86 & $1,73-2,00$ \\
\hline Cereais matinais & 2,86 & $2,49-3,24$ & 2,64 & $2,33-2,96$ & 2,54 & $2,15-2,92$ \\
\hline Bebidas alcoólicas & 6,99 & $6,56-7,42$ & 6,94 & $6,57-7,30$ & 7,44 & $6,88-8,00$ \\
\hline Total & 1,94 & $1,84-2,04$ & 1,93 & $1,83-2,02$ & 1,69 & $1,57-1,81$ \\
\hline
\end{tabular}

IC95\%: intervalo de 95\% de confiança. 
Tabela 5

Preço médio ( $\$$ \$1.000kcal) pago por grupos e subgrupos de alimentos definidos segundo natureza, extensão e o propósito do processamento aos quais eles foram submetidos, segundo área de localização do domicílio. Pesquisa de Orçamentos Familiares, Brasil, $2008-2009$.

\begin{tabular}{|c|c|c|c|c|}
\hline \multirow[t]{3}{*}{ Grupos e subgrupos de alimentos } & \multicolumn{4}{|c|}{ Preço dos alimentos (R\$/1.000kcal) } \\
\hline & \multicolumn{2}{|c|}{ Rural } & \multicolumn{2}{|c|}{ Urbana } \\
\hline & Média & IC95\% & Média & IC95\% \\
\hline In natura e minimamente processados (1) & 1,78 & $1,70-1,86$ & 2,37 & $2,30-2,44$ \\
\hline Arroz & 0,53 & $0,52-0,54$ & 0,53 & $0,53-0,54$ \\
\hline Feijão & 0,92 & $0,89-0,96$ & 0,99 & $0,97-1,01$ \\
\hline Carnes & 3,84 & $3,73-3,95$ & 4,47 & $4,36-4,59$ \\
\hline Leite & 2,01 & $1,91-2,11$ & 2,61 & $2,58-2,65$ \\
\hline Frutas & 3,65 & $3,42-3,88$ & 4,27 & $4,17-4,36$ \\
\hline Raízes e tubérculos & 2,03 & $1,87-2,19$ & 2,36 & $2,30-2,41$ \\
\hline Hortaliças & 10,17 & $9,87-10,46$ & 10,37 & $10,15-10,58$ \\
\hline Peixes & 6,53 & $5,97-7,08$ & 8,82 & $8,31-9,32$ \\
\hline Ovos & 3,42 & $3,28-3,56$ & 3,11 & $3,05-3,18$ \\
\hline Outros alimentos & 2,29 & $1,94-2,64$ & 3,24 & $3,03-3,45$ \\
\hline Ingredientes culinários processados (2) & 0,54 & $0,52-0,55$ & 0,66 & $0,64-0,69$ \\
\hline Açúcar & 0,28 & $0,28-0,29$ & 0,28 & $0,27-0,28$ \\
\hline Óleos vegetais & 0,35 & $0,34-0,36$ & 0,39 & $0,37-0,41$ \\
\hline Farinha de mandioca & 0,44 & $0,43-0,46$ & 0,48 & $0,47-0,49$ \\
\hline Farinha de trigo & 0,60 & $0,58-0,62$ & 0,55 & $0,53-0,56$ \\
\hline Macarrão & 0,90 & $0,87-0,92$ & 0,93 & $0,91-0,95$ \\
\hline Gordura animal & 1,34 & $1,25-1,44$ & 1,63 & $1,55-1,70$ \\
\hline Outros ingredientes & 4,13 & $2,93-5,34$ & 6,41 & $5,04-7,79$ \\
\hline In natura e minimamente processados e ingredientes culinários em conjunto $(1+2)$ & 1,16 & $1,12-1,20$ & 1,63 & $1,58-1,69$ \\
\hline Processados e ultraprocessados & 2,01 & $1,93-2,09$ & 2,47 & $2,40-2,54$ \\
\hline Processados & 3,24 & $3,00-3,48$ & 4,00 & $3,85-4,14$ \\
\hline Queijos & 3,55 & $3,36-3,75$ & 4,03 & $3,94-4,12$ \\
\hline Carnes processadas & 3,69 & $3,34-4,05$ & 3,88 & $3,63-4,14$ \\
\hline Conservas & 3,90 & $3,38-4,42$ & 5,34 & $4,94-5,75$ \\
\hline Ultraprocessados & 1,91 & $1,82-1,99$ & 2,33 & $2,26-2,40$ \\
\hline Pães & 1,41 & $1,37-1,45$ & 1,50 & $1,47-1,53$ \\
\hline Produtos doces ou sobremesas & 1,72 & $1,65-1,79$ & 2,04 & $1,98-2,10$ \\
\hline Produtos de panificação & 1,47 & $1,40-1,54$ & 1,69 & $1,65-1,73$ \\
\hline Confeitos e outros produtos à base de açúcar & 2,42 & $2,32-2,51$ & 2,59 & $2,52-2,67$ \\
\hline Salgadinhos e petiscos salgados & 1,36 & $1,31-1,41$ & 1,48 & $1,44-1,52$ \\
\hline Bebidas adoçadas & 5,22 & $5,02-5,42$ & 5,31 & $5,21-5,41$ \\
\hline Refrigerantes & 3,95 & $3,80-4,09$ & 4,19 & $4,10-4,28$ \\
\hline Outras bebidas & 12,99 & $11,74-14,24$ & 9,02 & $8,78-9,26$ \\
\hline Carnes ultraprocessadas & 2,07 & $2,01-2,14$ & 2,43 & $2,37-2,49$ \\
\hline Pratos ou refeições prontas & 2,55 & $2,38-2,72$ & 3,26 & $3,15-3,37$ \\
\hline Molhos e patês & 1,56 & $1,44-1,68$ & 1,74 & $1,67-1,80$ \\
\hline Cereais matinais & 2,08 & $1,81-2,35$ & 2,50 & $2,29-2,70$ \\
\hline Bebidas alcoólicas & 5,70 & $5,16-6,24$ & 6,91 & $6,65-7,17$ \\
\hline Total & 1,28 & $1,24-1,33$ & 1,88 & $1,81-1,94$ \\
\hline
\end{tabular}

IC95\%: intervalo de 95\% de confiança. 
evidências indicam que nas últimas décadas o preço dos alimentos tem variado de forma a favorecer o consumo dos alimentos processados e ultraprocessados, tanto no Brasil quanto em diversos outros países em desenvolvimento 26 . Portanto, na ausência de políticas públicas capazes de reverter as tendências atuais, a continuidade na redução do preço dos alimentos processados e ultraprocessados, em relação aos demais grupos, poderá criar ambiente ainda mais favorável à elevação de seu consumo nos próximos anos.

Mesmo que esse seja o primeiro estudo destinado a comparar o preço dos alimentos em diferentes estratos da população brasileira, levando em conta a natureza, intensidade e propósito do processamento dos alimentos, resultados semelhantes já podiam ser identificados em países desenvolvidos há quase uma década. A relação inversa entre a densidade energética dos alimentos e seu preço por caloria propõe explicação para o menor preço dos ingredientes culinários, assim como alguns subgrupos dos alimentos frescos, como cereais e leguminosas secas, e maior preço dos alimentos frescos como frutas, hortaliças e carnes frescas em relação aos demais grupos de alimentos 27,28,29.

A despeito do aumento geral dos preços verificado com aumento do nível de renda das famílias, o preço dos alimentos processados e ultraprocessados em relação ao conjunto dos demais alimentos se manteve estável, indicando cenário econômico semelhante para escolha de alimentos entre todas as classes de rendimentos. Esse achado não corrobora a evidência identificada em mercados desenvolvidos 12,15,30, segundo a qual indivíduos de menor renda sofreriam com cenário de preços ainda mais desfavorável à adoção de práticas alimentares do que aquele visualizado pela população como um todo. Uma vez que a alimentação possui o maior peso no orçamento das famílias mais pobres, sabe-se que a aquisição de alimentos por esse grupo populacional tende a ser especialmente sensível a variações no preço dos produtos 13 .

Os resultados desta pesquisa destacam a importância de medidas relacionadas ao preço dos alimentos na adoção de uma alimentação saudável. Políticas que resultem no aumento do preço dos alimentos processados e ultraprocessados, distanciando-os ainda mais do preço dos demais alimentos, são necessárias e ainda não aplicadas no Brasil. Tais medidas possuem grande propensão a conseguir resultados efetivos em curto prazo, visto que influenciam diretamente a escolha dos consumidores 31 . Cabe destacar que, no ano de 2013, o Governo Federal brasileiro buscou obter resultado semelhante (distanciar o preço de alimentos processados e ultraprocessados do preço dos demais alimentos) agindo na redução do preço dos alimentos in natura e minimamente processados ao invés de no aumento do preço dos alimentos processados e ultraprocessados. Essa tentativa consistiu de uma ação de desoneração dos produtos da cesta básica dos impostos federais 32 , e, embora essa medida não tenha tido como alvo específico apenas alimentos saudáveis, diversos alimentos in natura ou minimamente processados foram beneficiados 32 . Contudo, o impacto real dessa desoneração sobre o preço e o consumo dos alimentos se mostrou pouco efetiva ${ }^{33}$. Possivelmente, uma alteração significativa no consumo alimentar da população brasileira dependerá de medidas menos conservadoras, como a adoção de taxas sobre o consumo de alimentos ultraprocessados 34 .

Já que o preço de alimentos processados e ultraprocessados é reduzido potencialmente por causa da capacidade da indústria de ampliar sua produtividade e eficiência 8,22 , a criação de linhas especiais de crédito voltadas a avanços na cadeia de produção e distribuição de alimentos in natura ou minimamente processados seria também uma alternativa para promover a redução de seu preço 8,22 .

\section{Conclusão}

Os resultados do presente estudo expõem o preço por caloria dos grupos de alimentos no Brasil segundo classificação utilizada no Guia Alimentar para População Brasileira. Foram identificados contrastes de preço em relação à natureza propósito e intensidade do processamento dos alimentos. Enquanto alimentos frescos como carnes, leite, frutas e hortaliças tendem a custar mais caro que alimentos ultraprocessados, grãos secos (como o arroz e o feijão) despontam como uma alternativa mais econômica para a adoção de práticas alimentares saudáveis. 


\section{Colaboradores}

R. M. Claro contribuiu com a concepção e delineamento do estudo, análise e interpretação dos dados, redação e revisão do artigo. E. G. Maia contribuiu com a interpretação dos dados, redação e revisão do artigo. B. V. L. Costa e D. P. Diniz contribuíram com a interpretação dos dados e revisão do artigo.

\section{Agradecimentos}

Os autores agradecem à Coordenação de Aperfeiçoamento de Pessoal de Nível Superior (Capes) e à PróReitoria de Pesquisa da Universidade Federal de Minas Gerais (PRPQ-UFMG) pelo financiamento de bolsas de estudo.

\section{Referências}

1. World Health Organization. Preventing chronic diseases a vital investment. http://whqlibdoc.who. int/publications/2005/9241563001_eng.pdf (acessado em 10/Jun/2015).

2. Ng M, Fleming T, Robinson M, Thomson B, Graetz $\mathrm{N}$, Margono C, et al. Global, regional, and national prevalence of overweight and obesity in children and adults during 1980-2013: a systematic analysis for the Global Burden of Disease Study 2013. Lancet 2014; 384:766-81.

3. Monteiro CA, Moura EC, Conde WL, Popkin BM. Socioeconomic status and obesity in adult populations of developing countries: a review. Bull World Health Organ 2004; 82:940-6.

4. Instituto Brasileiro de Geografia e Estatística. Pesquisa de Orçamentos Familiares 2002/2003: análise da disponibilidade domiciliar de alimentos e do estado nutricional no Brasil. http://www.ibge.gov. br/home/estatistica/populacao/condicaodevida/ pof/2002analise/default.shtm (acessado em 10/ Jun/2015).

5. Instituto Brasileiro de Geografia e Estatística. Pesquisa de Orçamentos Familiares 2008/2009: antropometria e estado nutricional de crianças, adolescentes e adultos no Brasil. http://www.ibge.gov. br/home/estatistica/populacao/condicaodevida/ pof/2008_2009_encaa/default.shtm (acessado em 10/Jun/2015).
6. Malta DC, Duarte EC. Causas de mortes evitáveis por ações efetivas dos serviços de saúde: uma revisão da literatura. Ciênc Saúde Coletiva 2007; 12:765-76

7. World Health Organization. Diet, nutrition and the prevention of chronic diseases. http://whqlibdoc. who.int/trs/who_trs_916.pdf (acessado em 10/ Jun/2015).

8. Monteiro CA, Levy RB, Claro RM, Castro IRR, Cannon G. Increasing consumption of ultra processed foods and likely impact on human health: evidence from Brazil. Public Health Nutr 2011; 14:5-13.

9. Martins APB, Levy RB, Claro RM, Moubarac JC, Monteiro CA. Participação crescente de produtos ultraprocessados na dieta brasileira (1987-2009). Rev Saúde Pública 2013; 47:656-65.

10. Ministério da Saúde. Guia alimentar para a população brasileira. http://portalsaude.saude.gov.br/ images/pdf/2014/novembro/05/Guia-Alimentar -para-a-pop-brasiliera-Miolo-PDF-Internet.pdf (acessado em 06/Jun/2015).

11. European Food Information Council. The determinants of food choice. EUFIC Review 2005; (4). http://www.eufic.org/article/en/expid/reviewfood-choice/.

12. Irala-Estévez J, Groth M, Johansson L, Oltersdorf U, Prättälä R, Martínez-González MA. A systematic review of socioeconomic differences in food habits in Europe: consumption of fruit and vegetables. Eur J Clin Nutr 2000; 54:706-14. 
13. Claro RM, Carmo HCE, Machado FMS, Monteiro CA. Renda, preço dos alimentos e participação de frutas e hortaliças na dieta. Rev Saúde Pública 2007; 41:557-64.

14. Moubarac JC, Martins APB, Claro RM, Levy RB, Cannon G, Monteiro CA. Consumption of ultraprocessed foods and likely impact on human health. Evidence from Canada. Public Health Nutr 2013; 16:2240-8.

15. Monteiro CA, Moubarac JC, Cannon G, Ng SW, Popkin B. Ultra-processed products are becoming dominant in the global food system. Obes Rev 2013; 14 Suppl 2:21-8.

16. Monteiro CA. The big issue is ultra-processing. The price and value of meals. World Nutrition 2011; 2:271-82.

17. Ministério da Saúde. Plano de ações estratégicas para o enfrentamento das doenças crônicas não transmissíveis no Brasil (2011-2022). http://bvsms. saude.gov.br/bvs/publicacoes/plano_acoes_en frent_dcnt_2011.pdf (acessado em 08/Jun/2015).

18. Câmara Interministerial de Segurança Alimentar e Nutricional. Estratégia Intersetorial de Prevenção e Controle da Obesidade: recomendações para estados e municípios. http://www.ebah.com.br/ content/ABAAAgmF4AL/estrategia-intersetorialprevencao-controle-obesidade (acessado em 08/ Jun/2015).

19. Núcleo de Estudos e Pesquisas em Alimentação, Universidade Estadual de Campinas. Tabela Brasileira de Composição de Alimentos - TACO - versão 4.1. Campinas: Universidade Estadual de Campinas; 2004.

20. Agricultural Research Service, U.S. Department of Agriculture. USDA National Nutrient Database for Standard Reference. Release, 15. Beltsville: U.S. Department of Agriculture; 2002.

21. Monteiro C, Cannon G, Levy RB, Claro R, Moubarac J-C, Martins AP, et al. The big issue for nutrition, disease, health, well-being. http://www. wphna.org/htdocs/2012_dec_wn3_food_system. htm (acessado em 10/Jun/2015).

22. Monteiro CA, Levy RB, Claro RM, Castro IRR, Cannon G. A new classification of foods based on the extent and purpose of food processing. Cad Saúde Pública 2010; 26:2039-49.

23. Lagiou P, Trichopoulou A; DAFNE Contributors. The DAFNE initiative: the methodology for assessing dietary patterns across Europe using household budget survey data. Public Health Nutr 2001; 4:1135-41.
24. Trichopoulou A, Naska A; DAFNE III Group. European food availability databank based on household budget surveys: the Data Food Networking initiative. Eur J Public Health 2003; 13:24-8.

25. Monteiro CA. All the harmful effects of ultra processed foods are not captured by nutrient profiling. Public Health Nutr 2009; 12:1968.

26. Wiggins S, Keats S, Han E, Shimokawa S, Hernández JAV, Claro RM. The rising cost of a healthy diet: changing relative prices of foods in highincome and emerging economies. http://www. odi.org/sites/odi.org.uk/files/odi-assets/publi cations-opinion-files/9580.pdf (acessado em 08/ Jun/2015).

27. Monsivais P, Drewnowski A. Lower-energy-density diets are associated with higher monetary costs per kilocalorie and are consumed by women of higher socioeconomic status. J Am Diet Assoc 2009; 109:814-22.

28. Townsend MS, Aaron GJ, Monsivais P, Keim NL, Drewnowski A. Less-energy-dense diets of lowincome women in California are associated with higher energy-adjusted diet costs. Am J Clin Nutr 2009; 89:1220-6.

29. Waterlander WE, de Haas WE, van Amstel I, Schuit AJ, Twisk JW, Visser M, et al. Energy density, energy costs and income: how are they related? Public Health Nutr 2009; 13:1599-608.

30. Painter J, Rah J, Lee Y. Comparison of international food guide pictorial representations. J Am Diet Assoc 2002; 102:483-9.

31. Silva JG, Del Grossi ME, França CG, organizadores. Fome zero: a experiência brasileira. Brasília: Ministério do Desenvolvimento Agrário; 2010. (Série NEAD Especial, 12).

32. Brasil. Lei no 12.839 , de 9 de julho de 2013. Diário Oficial da União 2013; 10 jul.

33. Lima S. Alimentos e desconto na água continuam segurando inflação, mostra prévia. Folha de S. Paulo 2014; 18 jun. http://www1.folha.uol.com. br/mercado/2014/06/1472305-previa-da-infla cao-de-junho-fica-em-047.shtml.

34. Claro RM, Levy RB, Popkin BM, Monteiro CA. Sugar-sweetened beverage taxes in Brazil. Am J Public Health 2012; 102:178-83. 


\section{Abstract}

This study aims to describe the prices of food groups consumed in Brazil considering the nature, extent, and purpose of their processing. Data were obtained from the Brazilian Household Budget Survey for 2008 2009. The mean prices of the groups (natural, cooking ingredients, processed, and ultra-processed) and their respective food subgroups were estimated for Brazil according to income, region, and area. Natural products and cooking ingredients showed lower prices per calorie when compared to the other groups, suggesting an economic advantage to preparing meals at home when compared to replacing them with ultraprocessed foods. Families with the highest income paid the highest prices for their food, while families in the Northeast and North regions and rural areas paid the lowest. While fresh foods (meat, milk, fruit, and vegetables) tend to cost more than ultra-processed foods, dry grains (like rice and beans) are a more economical alternative for adopting healthy eating practices.

Food Habits; Food Handling; Socioeconomic Factors

\section{Resumen}

El estudio tuvo como objetivo describir el precio de los grupos de alimentos consumidos en Brasil, considerando la naturaleza, la extensión y el propósito de su procesamiento. Se utilizaron datos provenientes de la Investigación de Presupuestos Familiares de 2008-2009. Se estimó el precio medio de los grupos (in natura, ingredientes culinarios, procesados y ultraprocesados) y sus respectivos subgrupos de alimentos para Brasil según renta, región y área. Los productos in natura e ingredientes culinarios presentaron menor precio por caloría, cuando se comparan con los demás grupos, sugiriendo una ventaja económica en la preparación de comidas en el hogar, en comparación a su sustitución por alimentos ultraprocesados. Las familias de mayor nivel económico pagaron un precio más elevado por sus adquisiciones, mientras que las regiones Nordeste, Norte y el área rural pagaron menores precios. En lo referente a los alimentos frescos (como carnes, leche, frutas y hortalizas) tienden a costar más caro que los alimentos ultraprocesados, legumbres (como el arroz y los frijoles) despuntan como una alternativa más económica para adopción de prácticas alimentarias saludables.

Hábitos Alimenticios; Manipulación de Alimentos; Factores Socioeconómicos
Recebido em 30/Jun/2015

Versão final reapresentada em 11/Nov/2015

Aprovado em 17/Nov/2015 